\title{
EDUCACIÓN TECNOLÓGICA Y PROFESIONAL INTEGRADA A LA EDUCACIÓN BÁSICA EN PERSPECTIVA UNITARIA
}

\author{
Bruno Miranda Neves ${ }^{1}$ \\ Kerolin Tainá da Silva Camargo²
}

\begin{abstract}
Resumo
0 texto se propõe a apresentar conceitos centrais para a organização de práticas pedagógicas que integrem a Educação Tecnológica e Profissional à Educação Básica, com vistas ao pleno desenvolvimento das capacidades humanas dentro da perspectiva de garantia da dupla cidadania (social e econômica) nas sociedades contemporâneas. Por isso, nos dispusemos a expor as bases ético-políticas da Pedagogia Histórico-Crítica (PHC); algumas ideias chaves para organização do currículo integrado que supere a tendência hegemônica da Ideologia das Competências, e apontamentos teóricos e metodológicos para fundamentar projetos políticos pedagógicos que integrem trabalho, ciência, cultura e tecnologia para reunificação das dimensões manuais e intelectuais do trabalho.
\end{abstract}

Palavras-chave: Integração Formação Profissional-Educação Geral; Integração curricular; Pedagogia Histórico-Crítica; Economia da Educação; Métodos de Formação Profissional

\section{Resumen}

El texto se propone presentar conceptos centrales para la organización de prácticas pedagógicas que integren la Educación Tecnológica y Profesional a la Educación Básica, con vistas al pleno desarrollo de las capacidades humanas dentro de la perspectiva de garantía de la doble ciudadanía (social y económica) en las sociedades contemporáneas. Por eso, nos dispusimos a exponer las bases ético-políticas de la Pedagogía Histórico-Crítica (PHC); algunas ideas claves para la organización del currículo integrado que supere la tendencia hegemónica de la ideología de las Competencias, y apuntes teóricos y

\footnotetext{
1 Mestre em Educação pelo PPGEduc da UFRRJ. Doutorando na UERJ. E-mail: bmirandaneves@yahoo.com.br

${ }^{2}$ Graduada em Pedagogia pela UERJ. E-mail: kerolintainascamargo@gmail.com
} 
metodológicos para fundamentar proyectos políticos pedagógicos que integren trabajo, ciencia, cultura y tecnología para la reunificación de las dimensiones manuales e intelectuales del trabajo.

Keywords: Integración Formación Profesional-Educación General; Integración curricular; Pedagogía Histórico-Crítica; Economía de la Educación; Métodos de Formación Profesional.

\begin{abstract}
The text proposes to present central concepts for the organization of pedagogical practices that integrate Technological and Professional Education with Basic Education, with a view to the full development of human capacities within the perspective of guaranteeing dual citizenship (social and economic) in societies contemporary. For this reason, we were prepared to expose the ethical-political bases of Historical-Critical Pedagogy (HCP); some key ideas for organizing the integrated curriculum that overcomes the hegemonic tendency of the Ideology of Competences, and theoretical and methodological notes to support pedagogical political projects that integrate work, science, culture and technology to reunify the manual and intellectual dimensions of work.
\end{abstract}

Keywords: Professional Training-General Education integration; Curricular Integration; Historical-Critical Pedagogy; Education Economics; Professional Training Methods.

\title{
Introdución
}

La educación pública fue una conquista de la clase trabajadora, convergiendo, aunque de manera contradictoria, con los intereses del capital frente a los avances de las fuerzas productivas. Los logros en este campo tienden a ser apropiados por el conservadurismo de la clase dominante desde sus aspectos materiales (distribución desigual de la oferta educativa) hasta la hegemonía cultural (empleabilidad, emprendimiento, calidad total, etc.).

A lo largo del desarrollo capitalista, tuvimos diferentes formas de separación entre propósitos, conocimiento y medios de producción, refinando la división social y técnica del trabajo como instrumento de subsunción de los trabajadores al capital. Independientemente de la complejidad de lo que se haga, la fuerza laboral permanece atrapada como una mercancía especial. De allí, la pertinencia de abordar el derecho a la 
educación pública, gratuita, laica y universal para las clases históricamente excluidas del proceso de escolarización.

La reestructuración productiva que comenzó en la década de 1970 engendra una nueva cultura de trabajo, a través de la cual se renueva el sentido común, las demandas de la formación profesional y sus centros educativos. Las teorías e ideologías sobre la educación vinculadas al pensamiento liberal y neoliberal - Capital Humano, Pedagogía de las Competénciasy Ideología del Capital Social - han atribuido a la educación y la formación profesional el poder de mejorar la productividad, los ingresos y el bienestar ${ }^{3}$ (NEVES, 2015). Esto nos remite a algunas cuestiones: ¿por qué, para quién, cómo, con qué recursos, cuándo producimos etc.?

Las alteraciones provocados por la apropiación privada del avance tecnológico y la retirada de derechos, expresan la intensidad y la regresión de la crisis del capital y la nueva necesidad de un reconocimiento de la distribución desigual de las oportunidades educativas, condicionadas por las formas de reproducción global de vida material en la sociedad de clases.

En países de capitalismo dependiente, es aún más importante tener trabajo (las actividades económicas genéricas y concretas) como principio educativo, porque a través de la mejora de las potencialidades humanas, podemos luchar por la participación de cada individuo en el proceso de producción social de la existencia y en el disfrute de lo que es producido.

No estamos de acuerdo con el un currículo integrado bajo la inspiración de la pedagogía o la ideología de las competencias, ya que está dirigido y limitado por y para la utilidad del saber en la realización de acciones prácticas. Desde nuestro punto de vista, la integración es establecida por la historicidad de la producción material y por la generación de conocimiento que conforma áreas profesionales-tecnológicas y disciplinas científicas que se despliegana partir de los conceptos a ser enseñados. Los movimientos de educadores inspirados en prácticas educativas emancipatorias han estado defendiendo la creación de "un tipo

${ }^{3}$ Las críticas a estas tresideologíasfueron realizadas respectivamente por Frigotto (2006), Ramos (2006) y Motta (2012). 
único de escuela preparatoria (básica-media) conduzca al joven hasta los umbrales de elección profesional, formándolo así como una persona capaz de pensar, estudiar, dirigir o controlar quién dirige" (GRAMSCI, 2010, p. 49).

En la lucha por la superación del mundo de las necesidades, la educación debe cumplir el papel de reunificar el trabajo intelectual y manual, devolviendo a los seres humanos la posibilidad de un amplio desarrollo de sus capacidades espirituales y materiales, o sea, la omnilateralidad (PISTRAK, 2009; SHULGIN, 2013; GRAMSCI, 2010; NOSELLA, 1992).

Nuestras formulaciones se apoyan en Mészáros (2005), cuando este ratifica que las soluciones y el horizonte de la pedagogía socialista deben ir más allá de la escuela, ocupándose de contraponerse a la sociedad capitalista en todas sus prácticas educativas, reconociendo que ellas tienen en común la (con)formación de la fuerza de trabajo a la explotación.

Señalamos que la integración curricular (inscripta en la tradición de la escuela unitaria y la educación politécnica) es parte del surgimiento de un sujeto pleno y consiente de si y de la interdependencia de la vida en comunidad. Por esto, asumimos que la cooperación es la unidad ética dentro y fuera de la escuela.

\section{Propuesta de educación profesional y tecnológica integrada con la educación básica}

Recuperamos aquello que fue legado por Pistrak (2009): la educación debe capacitar a los estudiantes para vislumbrar los aspectos del mundo físico y social, al entender las contradicciones y mediaciones que componen la totalidad concreta. La oposición entre el trabajo manual e intelectual, la división social y la división técnica del trabajo son los orígenes de los diferentes tipos de educación, reflejando la separación entre la producción y la escuela. Tal división no está en consonancia con una sociedad en la cual la libertad, la responsabilidad, la igualdad y la cooperación orientan las relaciones humanas. 
La perspectiva práctica de laenseñanza integrada es parte de las luchas sociales por laabolicióndel capital. El compromiso pedagógico conlatransformacióndel modo de producción social de la existencia tiene dimensiones éticas y políticas que se expresan en la praxis. La manifestación de este compromiso se basa en la Pedagogía Histórico-Crítica (PHC):

La educación es entendida como el acto de producir, directa e intencionalmente, en cada individuo singular, la humanidad que es producida histórica y colectivamente por el conjunto de hombres. En otros términos, esto significa que la educación es entendida como mediación en el seno de la práctica social global(SAVIANI, 2008, p. 37).

Por este sesgo, la integración curricular se opone a "la lógica hegemónica del contenido liberal-pragmático, que busca subordinar los procesos formativos a las demandas específicas e inmediatas del mercado" mediante la formación fragmentada y parcial (ARAÚJO, 2013, p. 27). En estas páginas, los términos "Educación Politécnica", "Educación Tecnológica", "Escuela Unitaria", "Currículo Integrado", etc. son brevemente comentadas desde la perspectiva de la organización de la educación básica (primaria y secundaria), aunque se practican y son referencias para todos los niveles y modalidades de la educación.

Entendemos aquí, a la enseñanza integrada, como una propuesta identificada con el proyecto estratégico de la escuela unitaria, de base gramsciana, sin ser confundido con ella. Se trata de una formación humana que procura romper con las dicotomías generales y específicas, políticas y técnicas o la educación propedéutica y profesional, herencias de una concepción fragmentaria y positivista de larealidad humana (ARAÚJ0, 2013, p. 28, énfasisenel original).

Presentamos algunos conceptos importantes para el ejercicio de la educación profesional que se orientan a laformación humana emancipada, prestando atención a laconstrucción de lajusticia social y ambiental. Al principio, subrayamos tres metas que son simultáneamente una necesidad para que las clases subordinadas se emancipen:

1) Educación profesional integrada con la educación básica para complementar la y nunca reemplazarla, conel objetivo de elevar la escolaridad y continuar los estudios. 
2) Generación de itinerarios formativos de profesionalización y calificación de los trabajadores, en sintonía con las distintas especialidades y ocupaciones de las ramas productivas.

3) Políticas públicas de formación profesional intersectorial que promuevan la inclusión social a través de la articulación de políticas de desarrollo locales, regionales, nacionales y de cooperación de países históricamente espoliados (FRIGOTTO; CIAVATTA; RAMOS, 2005).

El imperativo ético-político para un proyecto político-pedagógico no es reducir la educación a los intereses del mercado. Este es el imperativo principal que tiene como desafíola democracia, la que lucha, con la participación de educadores, gestores, estudiantes y responsables de alumnos, de definir las bases, los medios, objetivos y evaluación de la aprendizaje.

El concepto de formación humana integral e integrada nos impele a superar la división socio-técnica del trabajo, capacitando a los estudiantes para actividades intelectuales y manuales, planeando y ejecutando actividades particulares, teniendo conciencia de que cada tarea es una fracción del proceso social de producción de la existencia.

Los términos formación integrada, formación politécnica y educación tecnológica también buscan responder, también a las necesidades del mundo del trabajo,permeados por la presencia de la ciencia y la tecnología como fuerzas productivas, generadoras de valor, fuentes de riqueza. Pero también, a lahumanidadrelegada a actividadesprecarias, subempleo, desempleo, pérdida de lazos comunitarios e identidadpropia (CIAVATTA, 2012, p. 85 citada por RAMOS, 2014).

La integración entre la educación tecnológica y profesional con la educación básica no se restringe a preparar a los estudiantes para el mercado de trabajo o para el nivel escolar posterior. Sino que, cada persona se realice en la medida de las posibilidades; el sentido de la formación integrada es la apropiación de las bases científicas, técnicas, culturales, éticas, estéticas y tecnológicas de la vida contemporánea. El primer sentido de la integración es:

[...] de naturaleza filosófica y expresa una concepción de la formación humana, con base en la integración de todas las dimensiones de la vida en el proceso educativo, con el objetivo de la formación omnilateral de los sujetos. 
Estas dimensiones son constituidas por el trabajo, la ciencia y la cultura. El trabajo comprendido como la realización humana inherente al ser (sentido ontológico) y como práctica económica (sentido histórico asociado con el modo de producción); la ciencia comprendida como los conocimientos producidos por la humanidad que posibilita el avance contradictorio de las fuerzas productivas; y la cultura, que corresponde a los valores éticos y estéticos que guían las normas de conducta de una sociedad (RAMOS, 2014, p. 87).

Más allá de "unir", "sumar", "articular" o "adicionar" saberes profesionales a la educación general. "Integrar" es construir un proceso formativo que dé cuenta de las dimensiones de la vida (trabajo, cultura, ciencia y tecnología), ampliando los horizontes de los trabajadores jóvenes y adultos para la construcción de nuevas relaciones humanas, cada vez más preocupadas, con valores éticos e igualitarios.

La educación integrada es, entonces, una forma de organizar la educación posibilitando a los sujetos jóvenes y adultos a la internalización de los conocimientos históricamente construidos, con los cuales tendrán las condiciones de producir y crear nuevas soluciones a los problemas enfrentados en la realidad concreta, procurando su inserción digna en la vida productiva y comunitaria.

\section{El trabajo como unidad de ciencia, tecnología y cultura}

Entendemos a la humanidad como conjunto de mujeres y hombres que actúan en el mundo, promoviendo cambios en medios naturales y sociales para satisfacer sus necesidades individuales y colectivas. Esta acción genera conocimientos que son transmitidos y reformulados para representar en el plano del pensamiento, aquello que fue hecho para mantener nuestras condiciones de supervivencia.

El trabajo es un elemento estructural y constante en toda sociedad (ya sea esclavo, feudal, estamental o capitalista desarrollado). A medida que el modo de producción se modifica para satisfacer las necesidades, es decir, la forma en que trabajan, los seres humanos cambian su manera de ser y relacionarse. 
Sin embargo, el individuo no nace sabiendo cómo producir su propia existencia, aprende cómo hacerlo, a través de interacciones sociales y anticipación mental para la resolución de sus problemas (teleología). Para Kosik (1976, p. 127), estas actitudes tornan al ser humano único porque "se origina de la naturaleza, es parte de la naturaleza y al mismo tiempo supera a la naturaleza", no queda limitado apenas a su existencia física, sino que modifica el medio, produciendo incluso, aunque ya tenga satisfechas sus necesidades.

El concepto de trabajo se refiere a la intervención humana sobre el entorno natural, la capacidad de imaginar y aplicar soluciones que satisfagan sus necesidades, además de transmitir estos saberes (de una manera más o menos estructurada) a las generaciones actuales y futuras a través del intercambio cultural. El conocimiento sistematizado sobre un área tecnológica particular organiza los campos de la ciencia y, por eso, se relaciona con las disciplinas escolares. Tal conocimiento se legitima a lo largo de la historia, como resultado de un proceso de la humanidad que busca la comprensión y el cambio del medio que habita.

La ciencia es entonces, parte de un saber sistematizado y presentado a través de conceptos que representan las relaciones de fuerzas que son condicionadas y aprehendidas de la realidad o fenómeno considerado. En este contexto, la ciencia implica concepciones y métodos que se transmiten por generaciones y al mismo tiempo pueden ser pensados y cuestionados durante ese tiempo, en la medida que construimos nuevos conocimientos y modificamos el entorno.

Según Ramos (2014), la cultura debe ser comprendida en el sentido más amplio posible, articulada con un conjunto de representaciones y comportamientos que constituyen el modo de vida de una población determinada. Una formación integrada no da solo la posibilidad de un conocimiento científico sistematizado, también vinculado a la cultura, es capaz de desarrollar en el individuo un pensamiento crítico y creativo sobre los patrones artísticos, culturales, sociales, históricos, etc. 


\section{El trabajo como principio educativo}

Tomamos el Trabajo como un principio educativo, en tres dimensiones: a) Grado del desarrollo de las fuerzas productivas y de la sociedad; b) Contextos productivos y calificaciones socioprofesionales; c) El trabajo pedagógico como un modo específico de trabajo (SAVIANI, 2007).

Es a través del trabajo que producimos nuestra existencia, satisfaciendo nuestras necesidades de supervivencia. El trabajo como práctica económica en la sociedad contemporánea impele la educación hacia el aspecto profesional, en tanto que, bajo la concepción de la educación omnilateral, la profesionalización prioriza la formación humana con valores que ayudan en la reflexión intersubjetiva e intrasubjetiva de los aspectos que caracterizan la producción y reproducción social de la vida material.

Si por la formación general las personas adquieren conocimientos que permiten comprender la realidad, en la formación profesional el conocimiento científico adquiere, para el trabajador, el sentido de fuerza productiva, traduciéndose en técnicas y procedimientos, a partir de la comprensión de los conceptos científicos y tecnológicos básicos. Eso le permitirá actuar de manera autónoma y consciente en la dinámica económica de la sociedad. (RAMOS, 2014, p. 92)

La producción de conocimiento (por la mediación del aula, de la investigación, del laboratorio, del trabajo de campo, de las prácticas, etc.) potencializa la construcción de autonomía intelectual intimamente relacionada con las soluciones de la vida cotidiana. Este proyecto de educación, tiene como objetivo hacer de la escuela creativa, forjada por el dinamismo entre el conocimiento de las clases subalternas y el espacio escolar, en un movimiento continuo e innovador en el ámbito material, social y cultural.

Es importante considerar las condiciones de vida de los estudiantes para que se satisfagan sus necesidades básicas de recursos para su aprendizaje. Transformando la educación integrada en un proceso en el cual, todos son capaces de participar y construir, recuperando la noción de escuela como lugar de memoria. 
El concepto de tecnología trae la comprensión del proceso histórico de producción científica y tecnológica, y el entendimiento de cómo se ha dado la apropiación social de este conocimiento, para la transformación de las condiciones naturales de vida y para la expansión de las capacidades y potencialidades de los sentidos humanos (NEVES, 2015). Esta forma de estructuración del currículo requiere la identificación de las tecnologías que están asociadas en la producción de un bien o servicio sin retener la especificidad de su aplicación en los sectores respectivos (RAMOS, 2014).

Guiada por el principio de la relación entre la parte y la totalidad, la propuesta del plan de estudios "se inspira en las siguientes preguntas: ¿Cómo podemos proporcionar comprensiones globales y totalizantes de la realidad, a partir de la selección de componentes y contenidos curriculares? ¿Cómo orientar la selección de contenidos en el currículo de formación integrada?" (RAMOS 2014, p. 97-98). Y así evidenciar, que los procesos singulares de producción pertenecen a una realidad económica, histórica, política, cultural, social y ambiental más amplia. Es necesario que los currículos se elaboren dentro del estudio de problemas de un área profesional en todas las dimensiones en las que está inserta. Construyendo así contenidos sistematizados en diferentes disciplinas y áreas de conocimiento.

Con estas preguntas enfatizamos que la integración del conocimiento en el currículo depende de una postura epistemológica, cada cual desde su lugar, pero que construye relaciones permanentes con el otro. Profesores de Química, Matemática, Historia, Portugués, etc. Pueden intentar pensar en su actuación no solo como profesores de formación general, sino también de formación profesional, siempre que el proceso de producción de sus respectivos campos profesionales se conciba desde la perspectiva de la totalidad y su historicidad. (RAMOS, 2014, p. 98)

También destacamos, como factor fundamental para la formación integrada la práctica curricular, el reconocimiento de saberes y la certificación profesional. Pues la práctica no solo es valorizada por su carácter formal, sino también por lo pedagógico, después de todo es una parte fundamental del proceso de integración del conocimiento teórico con la parte práctica. El reconocimiento de saberes y la certificación "presentan enormes desafíos para las instituciones educativas, una vez que 
históricamente se han distanciado de las culturas de los trabajadores" (RAMOS, 2014, p. 98).

Las alianzas entre las instituciones de enseñanza y formación profesional con el sector productivo, los sindicatos y las organizaciones no gubernamentales se vuelven indispensables, ya que estos procesos generan otras formas de comprensión del conocimiento, porque se articulan fuera de los espacios formales de educación, por esto, debemos investigar cómo producen los trabajadores, se apropian de sus conocimientos y del mundo del trabajo.

\section{Nociones para la implementación del plan de estudios integrado}

Señalamos algunas pautas para la organización curricular de la educación profesional. Inicialmente, abordaremos las estructuras para construir el proyecto político-pedagógico construido colectivamente, reflejando vívidamente las concepciones, principios, propósitos y reglas decididos y reconocidos por la comunidad involucrada. Esta construcción implica condiciones para la organización del trabajo pedagógico: tiempo para la planificación individual y colectiva; implementación y evaluación de propuestas; contacto con el ambiente de trabajo; acompañamiento de los campos de las prácticas, etc. (RAMOS, 2014, p. 99). Conviene recordar que para la PHC, la organización del trabajo pedagógico establecerá parámetros para la confección de currículos, proyectos pedagógicos y planes de trabajo docente-discente, etc. en los cuales:

La práctica social se establece [...] como el punto de partida y llegada de la práctica educativa. De ahí, un método pedagógico que parte de la práctica social, en el que el profesor y el alumno se encuentran igualmente insertos, pero ocupan diferentes posiciones, condición para que tengan una relación fecunda en la comprensión y referenciación de las soluciones de los problemas planteados por la práctica social. Depende de los momentos intermedios del método identificar los problemas planteados por la práctica social (problematización), disponer de las herramientas teóricas y prácticas para su comprensión y solución. (instrumentación) y viabilizar su incorporación como elementos integrales de la propia vida de los estudiantes (catarsis). (SAVIANI, 2008, p.37) 
El trabajo de los educadores debe promover la catarsis de un proceso de superación de la sincresis (de la visión o sensación caótica del todo) hasta la síntesis (entendimiento de la realidad, de la unidad de lo diverso) por la mediación del análisis (abstracción/ideas sobre determinaciones de los más simples/concretos a los más complejos/abstractos) (DA SILVA, 2018). Por esto, destacamos cómo la participación creativa de los educadores en las luchas sociales por la emancipación se fusiona con la práctica pedagógica:

Sostenemos que no son las técnicas y/o procedimientos los que definen las prácticas pedagógicas integradoras, sino la institución de un ambiente que favorece la actitud didáctica integradora en la conducción de los procesos de enseñanza y frente a los objetos de conocimiento, y a los sujetos del proceso de enseñanza y aprendizaje, considerando la necesaria ampliación permanente de la calificación humana, los contextos, locales y universal, en los que los sujetos están insertos, así como las condiciones concretas para su realización. (ARAÚJO, 2013, p. 53)

Una práctica más centrada en las instituciones educativas valoriza el carácter humano de la acción de educar, y así, desestructura la noción dualista que caracteriza la relación entre la educación profesional y la educación básica. Por esto, es fundamental que se articulen tiempos y espacios para que las actividades colectivas tengan lugar para maestros, técnicos, supervisores de pasantías, estudiantes, movimientos sociales, empresas, etc.

\section{Fundamentación de un proyecto político-pedagógico integrado}

Con base en los estudios de Ramos (2014), enumeraremos los fundamentos para la construcción de un proyecto político-pedagógico para la enseñanza integrada. Comenzando por la construcción colectiva, en la cual los educadores y estudiantes participan en la evaluación de las condiciones objetivas, la discusión de las finalidades y de los principios del proyecto escolar, así como la generación del documento que guiará el trabajo y su evaluación.

La construcción del proyecto político-pedagógico debe llevarse a cabo de forma democrática y participativa, colectivamente, incluso si hay 
divergencias de opinión en relación al proyecto, a partir de estas discusiones debe llevarse a cabo la expansión a los debates, lo que hará que todos los involucrados se sientan parte como productores y que tienen participación activa en las decisiones.

La voluntad colectiva no es lo mismo que la voluntad de la mayoría, ya que no es el número de personas que defienden o aprueban una idea lo que determina las elecciones, sino el entendimiento colectivo de que, en determinadas condiciones, ciertas decisiones dan más unidad al grupo social que otras. La validez de las propuestas se evalúa con esta referencia, eligiendo aquellas que pueden conducir a un trabajo colectivo organizado, pautado por la coherencia entre principios, propósitos, objetivos y métodos de acción. (RAMOS, 2014, p. 99)

En la realidad rebelde, difícilmente encontramos experiencias en las que se respeten los derechos de los educadores y los alumnos, sobre el tema de la definición del contenido y la forma de enseñanza. Como detalla Ramos (2014, p. 100):

Así mismo, pensamos que la primera providencia es la generación de tiempos y espacios de enseñanza para la realización de actividades colectivas. A veces puede ser pertinente celebrar seminarios y reuniones con invitados externos, como intelectuales y gestores de la educación, facilitando discusiones sobre concepciones y políticas, permitiendo a la comunidad escolar presentar problemas conceptuales y operativos, en una estrategia de participación de educadores y estudiantes con el tema Esto, sin embargo, no es suficiente. Realizar talleres, cursos y debates a nivel regional, reuniendo a varias escuelas, también es fructífero. Además, es esencial continuar con procesos más locales e interactivos, pues, los educadores deben darse cuenta, en diálogo entre ellos, de que un proyecto de esta naturaleza es necesario y posible en su escuela.

Es imprescindible que los gestores de los sistemas públicos de enseñanza y formación profesional establezcan medidas que creen confianza en los educadores, favoreciendo, entre otras medidas: concursos públicos; planes de carrera y salarios que valoricen la calificación permanente y la dedicación exclusiva; e infraestructura.

Con base en Ramos (2014) y Ciavatta (2005), reafirmamos nociones que pueden ser consideradas por las instituciones educativas al formular y reformular planeamientos estratégicos, proyectos político-pedagógicos y proyectos de cursos. 
Investigar la dinámica productiva del territorio, sus características tecnológicas y ocupacionales para enseñar los contenidos y las habilidades que se requieren para que los trabajadores obtengan empleo, sin limitarse al mercado de trabajo. Involucrar a centros de formación, estudiantes, familia y sociedad local: la institución debe apreciar las formas en que los estudiantes están en el mundo, construyendo alternativas de inserción profesional y social, presentando con transparencia las posibilidades de ocupación que realmente existen y las que son potenciales.

Tener en mente las condiciones materiales del individuo y proporcionar las condiciones didáctico-pedagógicas para la comunidad escolar. Por ejemplo:

[...]lasnecesidades de los estudiantes para cumplir su jornada de estudios en términos de movilidad, alimentación, ingresos mínimos para mantenerse y permanecer en la escuela; segundo, la existencia de instalaciones que ofrecen laboratorios, biblioteca, talleres, espacios de ocio, talleres donde aprender la teoría y práctica de disciplinas y proyectos en curso (RAMOS, 2014, p.102).

Transformar la formación integrada para que sea democrática, participativa y siempre en proceso de ajuste, fomentando el trabajo pedagógico interdisciplinario.

Requiere que los maestros estén abiertos a la innovación, los temas y las experiencias más adecuadas para la integración. Las ideas en curso en las escuelas son, por ejemplo, proyectos que articulan arte y ciencia; proyectos de iniciación científica; componentes curriculares destinados a comprender los fundamentos sociopolíticos del área profesional, entre otros. El estudiante debe tener horizontes de captura del mundo más allá de las rutinas escolares, los límites establecidos y normalizados de la disciplina escolar, para que pueda apropiarse de la teoría y la práctica que hacen del trabajo una actividad creativa, fundamental para el ser humano. (RAMOS, 2014, p.102)

La preservación de la memoria institucional y comunitaria en la que se inserte también sirve como un vínculo de respeto y apreciación con el compromiso de todos y cada uno, frente de las constantes demandas de cambios en los formatos, los tiempos y los contenidos de las prácticas educativas. Este camino contribuye a la autoestima y la autonomía de los sujetos e instituciones ante posibles malentendidos y presiones procedentes de intereses contrarios a la humanización. 


\section{Organización del plan de estudios por eje tecnológico}

La diversidad mediante la cual los cursos pueden asignarse a actividades profesionales que se incluyen en más de un área de especialización (procesos, productos, servicios, producción etc.) configura el enorme desafío de organizar denominaciones, fiscalizar y supervisar, tanto lugares de trabajo como centros de formación de interés público. Refiriéndose al caso brasileño, Bastos (1998) afirma que

La implementación del eje tecnológico prevé coherencia con la especificidad de la educación profesional, que tiene su objeto de estudio e intervención centrada en la tecnología. Es a través del registro, la sistematización, la comprensión y el uso de los conceptos de tecnología, histórica y socialmente construidos, como elementos de enseñanza, investigación y extensión que la educación profesional y tecnológica se asume como un instrumento de innovación y transformación de las actividades económicas, pudiendo contribuir a la formación de los ciudadanos y al desarrollo del país. (BASTOS, 1998 citado por RAMOS, 2014, p. 103)

Identificar qué comprensión preside un conjunto de tecnologías que apoyan la producción, a través del rescate de la lógica del desarrollo económico, científico y tecnológico que se despliegan en el conocimiento es una de las formas posibles para la organización

Aunque existen diferentes conceptos de tecnología, algunos aspectos son recurrentes en las definiciones encontradas. La tecnología siempre modifica una realidad mediante el uso de un conjunto complejo de conocimiento tecnológico acumulado, transformando tanto la base técnica como las relaciones humanas. Como objeto de estudio, la tecnología puede entenderse como una "ciencia transdisciplinaria de las actividades de producción humana, el uso de objetos técnicos y hechos tecnológicos. El punto de vista de la escuela es la disciplina que estudia el trabajo humano y sus relaciones con los procesos técnicos. (MACHADO, 2008 citada por RAMOS, 2014, p.104)

Las instituciones y los educadores necesitan bases teóricas y metodológicas para que los saberes se tornen "accesibles [para el alumno] ya que son capaces de comprender los principios generales en los que se basa la multiplicidad de procesos y técnicas fundamentales para los sistemas de producción" (RAMOS, 2014, p. 104).

La enseñanza basada en estas nociones promueve la comprensión del proceso histórico de producción tecnológica y científica y hace entender cómo se producen las apropiaciones sociales de los conocimientos que 
transforman las condiciones naturales de la vida y expanden las capacidades, los sentidos y las potencialidades del ser humano. Es decir, asumir el trabajo como una condición humana de modificación del medio como el principio que guiará todo el proceso formativo.

Estructurar el currículo de esta forma exige la identificación de tecnologías específicas para la producción de un bien o servicio. En tanto, los ejes tecnológicos pueden transversalizar los currículos, sustentando las características que identifican cada uno de sus cursos.

\section{La totalidad como una perspectiva teórico-metodológica}

Cuando percibimos que los fenómenos son parte de un todo estructurado, vislumbramos las relaciones entre las partes y el todo. Con el propósito de enseñar, la elección de los componentes y contenidos curriculares adecuados, nos posibilita a establecer relaciones que aprendidas en el plano teórico, constituirán estudios de las esencias de los fenómenos a ser enseñados.

Optamos por la metodología según la cual "desde el conocimiento en su forma más contemporánea [...] se puede comprender la realidad y la propia ciencia misma en su desarrollo histórico", esto porque, el estado actual del conocimiento nos revela la construcción completa de su campo epistemológico y productivo (RAMOS, 2014, 107). A medida que nos aproximamos a la integración, los problemas originados en un área productiva, podemos estructurar los estudios de sus múltiples dimensiones, en base a "cuestiones específicas, se puede necesitar conocimientos generales y, por lo tanto, aprehenderlos para diversos fines más allá de los que motivaron su aprehensión" (RAMOS, 2014, 108).

Aunque la identidad epistemológica de cada contenido curricular/disciplinar trae consigo la profundidad científica que lo caracteriza, al relacionar parte y totalidad, es impresindible tener en cuenta el principio organizador de la interdisciplinariedad. Una vez que, "los 
conceptos de varias disciplinas serían relacionados a la luz de preguntas concretas que se pretende comprender" (RAMOS, 2014, p. 109).

\section{Repensar el lugar de la pasantia en la organización curricular}

Para no confundir la actividad intencional y supervisada de prácticas, con entrenamientos en servicios o "trainning", como concepción hegemónica de este componente curricular, debemos pensar la práctica o pasantía como "un momento de formación orientada y supervisada necesariamente prevista en el proyecto del curso", porque es en sí mismo una aproximación a las prácticas profesionales de manera simulada, de modo que se tenga una contextualización por intermedio de la práctica (RAMOS, 2014, p. 110).

Si bien este componente curricular no es obligatorio para todos los cursos técnicos, es deseable que las propuestas pedagógicas lo prevean, observando que la relación del estudiante que realiza la pasantía con la empresa que lo recibe, es diferente de la establecida con los empleados que venden su fuerza laboral.

Entendemos que "el desarrollo social y productivo construido a partir y por medio del trabajo es el vínculo integrador y sustentador de la capacidad humana para crear su propia historia de vida" (RAMOS, 2014, p.

112). Desde esta perspectiva, alentamos la pasantía

[...] no solo en su aspecto formal, sino principalmente por su carácter pedagógico, mientras que el reconocimiento de saberes y la certificación presentan enormes desafíos para las instituciones educativas, ya que implican la comprensión de otras formas de sistematización del conocimiento que operan fuera de los procesos productivos formales(RAMOS, 2014, p. 114).

Las formas interesantes de articular las observaciones y experiencias productivas de los estudiantes son a través de actividades de iniciación científica, extensión y monitoreo, siempre que esto esté dentro de la planificación del curso, como una posibilidad de "investigar cómo los trabajadores producen sus vidas, cómo se apropian de los lenguajes del mundo del trabajo y cómo traducen sus conocimientos" (RAMOS, 2014, p. 111). 


\section{La investigación como principio pedagógico articulado a la producción de conocimiento}

Para proporcionar a las personas conceptos que les permitan comprenderse a sí mismos, si está en el mundo y, por lo tanto, actuar en él a través del trabajo, transformando la naturaleza de acuerdo con las necesidades inmediatas, al mismo tiempo que comprende los conceptos fundamentales para que pueda cuidar la preservación frente a las necesidades de otros seres humanos y generaciones futuras.

La práctica de la investigación puede promover la creatividad y contribuir a la construcción de la autonomía intelectual, una vez tratada inseparablemente de la enseñanza, y estar vinculada al estudio para resolver los problemas teóricos y prácticos de la vida.

Por lo tanto, sostenemos que el concepto de formación humana integral sugiere superar al ser humano históricamente dividido por la división social del trabajo entre la acción de ejecutar y la acción de pensar, dirigir o planificar. El trabajo, la ciencia, la tecnología y la cultura forman una unidad, inseparable de la formación humana, con el trabajo como categoría central en sus dimensiones ontológicas e históricas. De esto se desprende el trabajo como principio educativo para guiar un proyecto de educación profesional comprometido con la formación humana. La investigación, a su vez, es un principio pedagógico. Este principio está estrechamente relacionado con el trabajo como principio educativo, que, cuando se asume en una educación integrada, contribuye a la formación de sujetos autónomos que pueden comprenderse a sí mismos en el mundo y así actuar en él a través del trabajo (RAMOS, 2014, p.94-95).

A través de la investigación, las reflexiones son posibles más allá del sentido común, causando curiosidad y pensamiento crítico y estructurado. Generando así una inquietud que es capaz de cuestionar la realidad, y que por lo tanto contribuye al tema, puede buscar respuestas en entornos de trabajo y producciones académicas.

Además, es esencial que esta (re) producción de conocimiento se guíe por un sentido ético. Por lo tanto, es esencial mejorar una concepción de la investigación, aplicada o no, así como el desarrollo científico y tecnológico comprometido con la producción de conocimiento, bienes y servicios que tengan como objetivo mejorar las condiciones de la vida colectiva y no solo producir bienes. para fortalecer el mercado y privilegiar el valor de cambio sobre el valor de uso, concentrando la riqueza y ampliando la brecha entre lo incluido y lo excluido. (RAMOS, 2014, p. 94) 
Enestaacepción, es necesario fomentar elfortalecimiento entre lainvestigación y ladocencia, bajo la mirada de contribución para laautonomía intelectual delsujeto, no solo por los aspectos deltrabajo, sino también por laconstrucción de otrasprácticassociales. Y asíavanzarenotros aspectos, para eldesarrollo de ideas y la participación ética de todos.

\section{Consideraciones finales}

El modo de producción capitalista realiza divisiones sociales y técnicas del trabajo, disociando los aspectos manuales e intelectuales y actualiza los diversos perfiles y ramas profesionales, utilizando una fuerza laboral con múltiples cualificaciones en interés de la acumulación.

La integración curricular se basa en los principios ético-políticopedagógicos del trabajo, la ciencia, la tecnología y la cultura, con el objetivo de reunir el trabajo y la educación en su esencia humanizadora. Desde este punto de vista, la lucha política, ética y pedagógica para materializar una educación politécnica (o tecnológica) para la formación omnilateral tiene una perspectiva histórica que no se limita a la escuela, y, por eso abarca la totalidad de las prácticas sociales - empezando por el intercambio material.

Observamos que el trabajo pedagógico realizado de manera colectiva y responsable desempeña un papel formativo sobre los educadores, frente a sus prácticas y la resignificación de sus saberes disciplinares, parciales y específicos a favor del entendimiento de las diferentes manifestaciones del trabajo en la sociedad contemporánea.

\section{Referencias}

ARAÚJO, Ronaldo Marcos de Lima. Práticas pedagógicas e ensino integrado. [Texto elaborado para discussão com os professores do IFPR. Parte integrante das atividades de pós-doutoramento realizado no Programa de Pós-Graduação em Políticas Públicas e Formação Humana PPFH - UERJ, no ano de 2013].

CIAVATTA, Maria. A formação integrada: a escola e o trabalho como lugares de memória e identidade. In: FRIGOTTO, Gaudêncio; CIAVATTA, Maria; RAMOS, Marise. Ensino Médio Integrado: concepções e contradições. São Paulo: Cortez, 2005. p. 93-106. 
DA SILVA, Livaldo Teixeira. Didática Coerente com a Pedagogia

Histórico-Crítica: elementos de aproximação da Educação do Jovem Adulto Trabalhador. Rio de Janeiro: RPC Editora, 2018.

FRIGOTTO, Gaudêncio. A produtividade da escola improdutiva. São Paulo: Cortez, 2006.

FRIGOTTO, Gaudêncio; CIAVATTA, Maria; RAMOS, Marise. Ensino Médio Integrado: concepções e contradições. São Paulo: Cortez, 2005.

GASPARIN, João Luiz; PETENUCCI, Maria Cristina. Pedagogia HistóricoCrítica: da teoria à prática no contexto escolar. Online. 2008. Disponível em: <http://www.diaadiaeducacao.pr.gov.br/portals/pde/arquivos/22898.pdf>. Acesso em 10 fevereiro 2019.

GRAMSCI, Antonio. Cadernos do cárcere, v.2. Rio de Janeiro: Civilização Brasileira, 2010.

KOSIK, Karel. Dialética do concreto. Rio de Janeiro: Paz e Terra, 1969.

MÉSZÁROS, István. Educação para além do capital. São Paulo: Boitempo, 2005.

MOTTA, Vânia Cardoso da. Ideologia do capital social: atribuindo uma face mais humana ao capital. Rio de Janeiro: EdUERJ, 2012.

NEVES, Bruno Miranda. O PROEJA no Colégio Pedro II: formação e qualificação docente em questão. Rio de Janeiro: RPC Editora, 2015.

NOSELLA, Paollo. A Escola de Gramsci. Porto Alegre: Artes Médicas: 1992.

PISTRAK, MoiseyMikhaylovich (Org.). A comuna escolar. São Paulo: Expressão Popular, 2009.

RAMOS, Marise Nogueira. A pedagogia das competências: autonomia ou adaptação? São Paulo: Cortez, 2006.

RAMOS, Marise Nogueira. História e política da educação profissional. Curitiba: Instituto Federal do Paraná, 2014.

SAVIANI, Demerval. História das ideias pedagógicas no Brasil. Campinas, SP: Autores Associados, 2008.

SAVIANI, Demerval. Trabalho e Educação: fundamentos ontológicos e históricos. Revista Brasileira de Educação, v. 12, n. 34, p. 152-180, 2007. 
SHULGIN, Viktor NikholaeVich. Rumo ao politecnismo (artigos e conferências). São Paulo: Expressão Popular, 2013. 